\title{
Another Way to Look at Donor Harvesting Effects with FUE
}

\author{
Paul T. Rose, MD, JD, FISHRS Coral Gables, Florida USA paultrose@yahoo.com
}

An important consideration of the FUE process is assessing the amount of wounding that can be done to the donor area and the number of grafts that can be removed before density diminishes to a point where evidence of the procedure is apparent. ${ }^{1}$

Many FUE surgeons suggest that $40-50 \%$ of the donor area follicular units (FUs) can be taken before there is a significant change in apparent density. The author differs with this calculation and seeks to demonstrate an important way to look at the potential wounding and thinning to the donor area with FUE.

When a physician examines densitometry photographs from the donor area, centrally and laterally he or she can observe that taking 1 FU with the intention of leaving adjacent FUs unharvested, leaves approximately 6 or 7 FUs surrounding the FU originally taken (Figure 1 ). This means, ideally, that the physician would want to leave the other adjacent FUs unharvested to be able to avoid a $2 \mathrm{FU}$ void. These numbers can certainly vary. It may be 1 out of 6 or 1 out of 8 in a "virgin" scalp, but review of numerous density images suggest that 1 out of 7 is most common with a density of 70-80 FUs/ $\mathrm{cm}^{2}$. From a mathematical perspective, this means that approximately $14 \%$ of the FUs can be obtained without an adjacent FU being harvested.

Why is this so important? If you assume that the safe donor area is between approximately 160 -

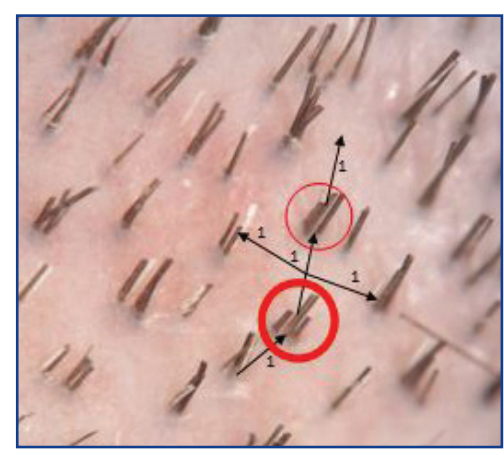

Figure 1. In planning to remove an $\mathrm{FU}$, it is important to note that each FU is usually surrounded by another adjacent 6-7 FUs. Extraction of more than about 1 out of 7 of the FUs makes it difficult to avoid taking adjacent FUs in large sessions. Also note inter-follicular distances.

$200 \mathrm{~cm}^{2}$ based on the calculations of Unger ${ }^{2,3}$ and then Cole, ${ }^{3,4}$ respectively, this means that a person with an average FU density of $80 \mathrm{FUs} / \mathrm{cm}^{2}$ would have available 13,000-16,000 total FUs. Of that, $14 \%$ would be 1,800-2,200 grafts without needing to harvest an adjacent graft.

If a subsequent session is undertaken, the surgeon would have significantly fewer grafts to choose from and there would be marked difficulty trying to find a location where adjacent FUs were not harvested. If adjacent FUs are harvested, then often a space of at least $6 \mathrm{~mm}^{2}$ would be evident (Figure 2). This assumes an interfollicular distance of usually $1 \mathrm{~mm}$. Taking an FU with a $1 \mathrm{~mm}$ punch usually produces a $1 \mathrm{~mm}$ or more diameter wound. As the FU is surrounded by two adjacent FUs, that leaves at least $3 \mathrm{~mm}$ of alopecic skin in one direction and often times more than $2 \mathrm{~mm}$ in width in the other direction. This is in part due to the fact that FUE scars can heal larger than the original punch.

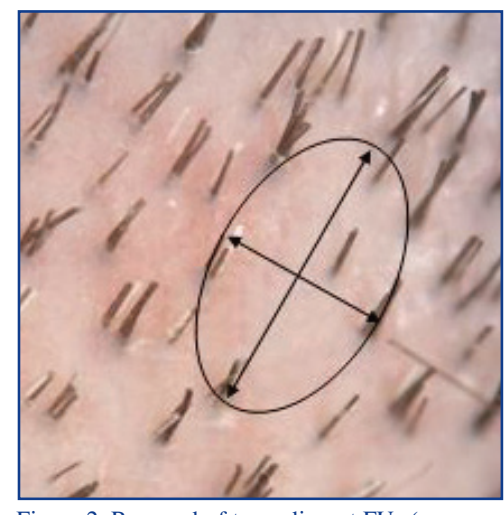

Figure 2. Removal of two adjacent FUs (compare to Figure 1) creates an area of hairless skin that can often be $6 \mathrm{~mm}^{2}$ or more. Such areas would likely be easily visible if the patient shaves his head. This measurement assumes an inter-follicular distance of approximately $1 \mathrm{~mm}$.
Even if a small punch of perhaps $0.7 \mathrm{~mm}$ is used, this can create a smaller initial wound but the area left behind from the punch still can leave a substantial area of alopecic skin. Again, it is important to note that FUE wounds can heal with a wound size in excess of the initial punch.

The increase in wound size may be due to the fact that with numerous punches the usual contraction that would occur with second intention healing is reduced due to changes in contractile forces dispersed over a large area. Also, at times the approach in aligning the punch may involve an attack angle that produces an oval shape as the inferior aspect of the punch touches the skin before the superior aspect. Sometimes this occurs as the surgeon tries to adjust for the angle change of the hair as it exits the skin as opposed to the angle of the hair within the skin. ${ }^{5,6}$ Exerting traction on the skin in an effort to straighten the hairs of the FUs while harvesting can also create an oval defect with a round punch, as a longer axis is created vertically. The oval produced is larger than the circle that would be formed by a perpendicular insertion of the punch. Consider that area of a circle is equal $\pi r^{2}$ $\left(1 \mathrm{~mm}\right.$ punch $\left..785 \mathrm{~mm}^{2}\right)$ versus an oval pi multiplied by length $\times$ width/4. $\left(1.2\right.$ (due to traction) $\left.\times 1.0 \times 3.14 / 4=.942 \mathrm{~mm}^{2}\right)$.

If a major aspect of the fervor for FUE is that the patient can wear his or hair quite short without evidence of the surgical procedure, then it seems that having areas of alopecic skin of $6 \mathrm{~mm}^{2}$ or more throughout the scalp would be counter to this supposed value of FUE. In view of the fact that surgeons are reporting performing cases of 5,000 or more grafts with FUE in the scalp, it seems obvious that many patients would lose a supposedly crucial benefit of the FUE process. In taking such large total amounts of FUs, these surgeons must be creating numerous areas of bald skin exceeding $6 \mathrm{~mm}^{2}$. A patient could hardly wear a short crew cut with such wounding. This is without considering that the physician must go beyond the "safe area" to achieve such numbers of grafts.

Also, the physician must be cognizant that patients with finer hair, low-hair density, and a lack of 3- and 4-hair FUs are at significant risk to have an appearance of thinning hair. Furthermore, the aging process can produce smaller caliber hairs with time and hairs within a follicular unit can be lost. This may further impact the perception of hair density in the scalp.

The figure of $40-50 \%$ has been mentioned as a percentage of hair that can be removed from an area before thinning is perceived. This seems to be an inappropriate use of the work of Dr. Emanual Marritt and not relevant to FUE surgery. While Marritt did report this percentage number, his work was based on plucking individual hairs not on taking follicular units. His work was also done with long hair not short hair. ${ }^{7}$

A physician might ameliorate some of the situation of prominent wounds by using partial FU removal, a technique that Cole and Rose referred to as Follicular Isolation Technique (FIT). ${ }^{8}$ Lorenzo also has been a proponent of a method that takes partial FUs. The use of scalp micro-pigmentation could also be used to camouflage the donor area. ${ }^{9}$ Devices to try to decrease the eventual wound size may also be helpful. ${ }^{10}$

It is this author's opinion that, while FUE has significant attributes, the effects of thinning and wound scar creation should be adequately discussed with the patient before endeavoring to perform the procedure. The surgeon must be aware of the limitations of the technique and the limitations of donor supply to optimize the patient's results and satisfaction with the hair restoration process. 
Editor's note: Dr. Rose makes very valuable points in this paper about the limits of donor harvesting with FUE. As I have thought about this same issue, I believe we need to think of applying the "illusion of density" principle that is widely used in describing how much hair must be transplanted into an area of bald skin to reach the point that the hair does not appear to be thinning. This threshold is often cited as the need to restore 50\% or more of original density. Hair caliber, texture, skin/hair color contrast, and average hairs per FU and in particular hair length have a significant impact on whether the 50\% threshold does in fact create the illusion of density. When patients shave their transplanted recipient area rarely does the transplanted area appear to be as dense as the adjacent/non-transplanted scalp. The hair has to be worn longer for the difference in density to not be apparent.

The same is true when we apply this principle to FUE harvesting. With low levels of harvesting with punches $0.8-0.9 \mathrm{~mm}$, many patients can shave their donor areas without the harvesting being evident. But as more and more grafts are harvested, a point will be reached that the patient must no longer shave the head if they wish the harvesting to not be apparent to others. They have to start wearing their donor area hair longer. For some, 2-3mm may be all that is needed, but for others, $1 \mathrm{~cm}$ or more will be needed. The longer hair will be necessary to have the "illusion of density" in the donor region. This is exactly what I explain to patients in giving informed consent for FUE. When they ask how many grafts can be extracted before they reach this threshold, I tell them it will vary from patient to patient because of variations in hair texture and caliber, skin to hair color contrast, donor density and average hairs per FU, and the healing of the extraction sites. Some patients may reach this threshold at 3,000-4000 grafts, others may go 1,000-2000 extractions higher before shaving the donor area is no longer possible without revealing evidence of surgery. I think the biggest problems happen with these mega-sessions of $4,000^{+}$extractions in an initial session.
Even though their surgeon very well may not have advised them of such, many of these patients will have already reached the point of needing to wear their donor hair longer in a single step. - RT

\section{References}

1. Rassman, W.R., et al. Follicular unit extraction; minimally invasive surgery for hair transplantation. Dermatol Surg. 2002; 28:720-728.

2. Unger, W. The Donor Site. In: W. Unger, ed. Hair Transplantation, 3rd Edition. New York: Marcel Dekker Inc.; 1995, pp. 183-212.

3. Devroye, J. An Overview of the Donor Area: Basic Principles. In: W. Unger, et al. eds. Hair Transplantation, 5th Edition. New York: Informa; 2011, pp. 257-259.

4. Cole, J. Donor Area Density Differences in Asians and Caucasians. Presented at the 17th Annual Meeting of the ISHRS, 2009; Amsterdam.

5. Zontos, G., P.T. Rose, and G. Nikiforidis. A mathematical proof of how the outgrowth angle of hair follicles influences the injury to the donor area in FUE harvesting. Dermatol Surg. 2014; 40(10):1147-1150.

6. Rose, P.T. Internal Angle of Hair Growth versus Exit Angle as It Relates to FUE/FIT. Presented at the 18th Annual Meeting of the ISHRS, October 20-24, 2010; Boston, Massachusetts, USA.

7. Marritt, E. The death of the density debate. Derm Surg. 1999; 25(8):654-660.

8. Rose, P.T., and J. Cole. Follicular Isolation Technique (FIT). Presented at the Aegean Masters Meeting; June 5-7, 2003; Greece.

9. Rassman, W.R., et al. Scalp micropigmentation. J of Clin Aesthet Dermatol. 2015; 8(3):35-42.

10. Rose, P.T. The Use of a Suction Apparatus to Improve Healing of FUE Wound Sites. Presented at the 21st Annual Meeting of the ISHRS, October 22-26, 2013, San Francisco, California, USA.

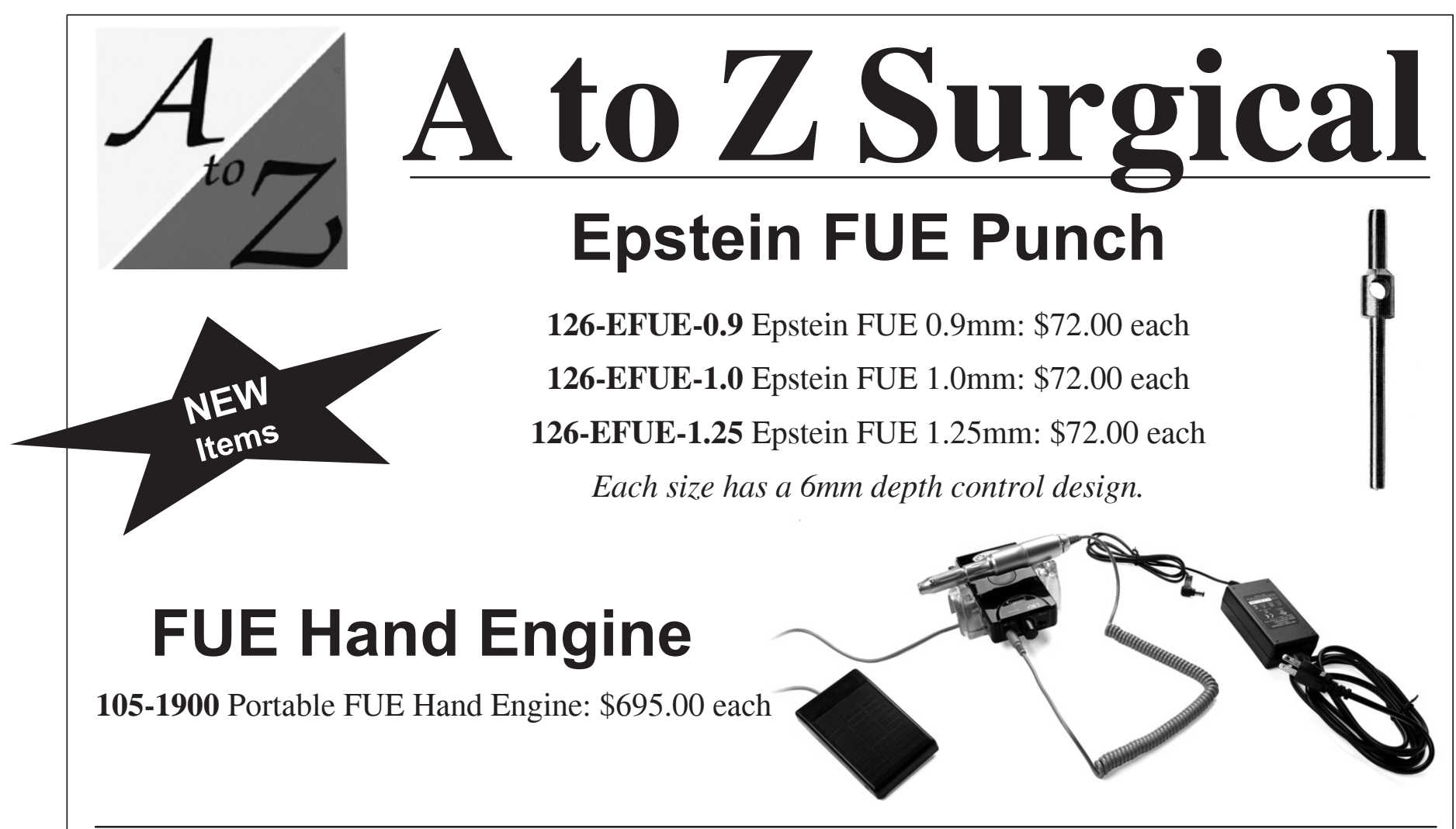

Phone: (800) 843-6266 • www.GeorgeTiemann.com • Fax: (800) 577-6050 